\title{
Entropy of never born protein sequences
}

\author{
Grzegorz Szoniec ${ }^{*}$ and Maciej J Ogorzalek
}

\begin{abstract}
Background: A Never Born protein is a theoretical protein which does not occur in nature. The reason why some proteins were selected and some were not during evolution is not known. We applied information theory to find similarities and differences in information content in Never Born and natural proteins.

Findings: Both block and relative entropies are similar what means that both protein kinds contain strongly random sequences.

An artificially generated Never Born protein sequence is closely as random as a natural one.

Conclusions: Information theory approach suggests that protein selection during evolution was rather random/ non-deterministic.

Natural proteins have no noticeable unique features in information theory sense.
\end{abstract}

Keywords: Never born protein, Block entropy, Relative entropy, Kullback-Leibler divergence, Origin of life

\section{Introduction}

Existing and known proteins are only a small subset of all possible sequences. Why were only some proteins selected during evolution? The reason is not known but two possible ways are considered: deterministic and random. To investigate theoretical sequences of amino acids a term Never Born Protein was introduced (Chiarabelli et al. 2006). Since 2006 only a few papers about them have been published. The most significant research has shown that $20 \%$ of them fold (i.e. reach stable and functional 3D structure) in laboratory conditions (Chiarabelli et al. 2006) and a tool for generating sequences with no similarity to natural proteins has been developed - Random Blast (Evangelista et al. 2007). The high folding ratio has been positively surprising and has abated opinion that existing proteins are the only stable and folding sequences. Surprisingly, as 1 out of 5 absolutely randomly generated proteins was a possibly useful one for living organisms. The authors did not expect so high percentage, furthermore their results came with doubts about correctness of their approach/methodology. Up to now this has been the most important discovery in Never Born protein science.

The question about proteins origin is still open. There are papers that proved natural and synthetic (random)

\footnotetext{
* Correspondence: myszonie@cyf-kr.edu.pl

Information Technology Department, Faculty of Physics, Astronomy and Applied Computer Science, Jagiellonian University, 4 Reymonta Street,
} Cracow 30-059, Poland only slightly different from each other(Weiss et al. 2000), there are also papers that proved these two groups of proteins are significantly different and protein selection during evolution was a driven process (Munteanu et al. 2008; De Lucrezia et al. 2012). The results depend on methodology (which is not discussed here because of limited scope of this report) nevertheless another open question is which approach should be considered as more correct and reliable than others.

Information theory (Shannon 1948) is applied to almost any branch of science. Information quantities i.e. Shannon entropy H (Shannon 1948) and Kullback-Leibler divergence (or relative entropy) $D_{\mathrm{KL}}$ (Kullback \& Leibler 1951) are defined as

$$
\begin{aligned}
& H=-\sum_{i} p_{i} \log \left(p_{i}\right) \\
& D_{K L}(P \| Q)=\sum_{i} p_{i} \log \left(p_{i} / q_{i}\right)
\end{aligned}
$$

Where $\mathrm{P}$ and $\mathrm{Q}$ are probability densities, $\mathrm{P}=\left\{\mathrm{p}_{\mathrm{i}}\right\}$ and $\mathrm{Q}=\left\{\mathrm{q}_{\mathrm{i}}\right\}$. Shannon entropy is a measure of uncertainty in an outcome with probability $p_{i}$ and relative entropy is a measure of similarity between two probability densities (it is not a true metric i.e. $\mathrm{D}_{\mathrm{KL}}(\mathrm{P} \| \mathrm{Q})$ is not equal to $\mathrm{D}_{\mathrm{KL}}$ $(\mathrm{Q} \| \mathrm{P})$ except when $\mathrm{P}=\mathrm{Q})$.

In protein science, information properties of natural proteins were comprehensively studied (Strait \& Dewey 


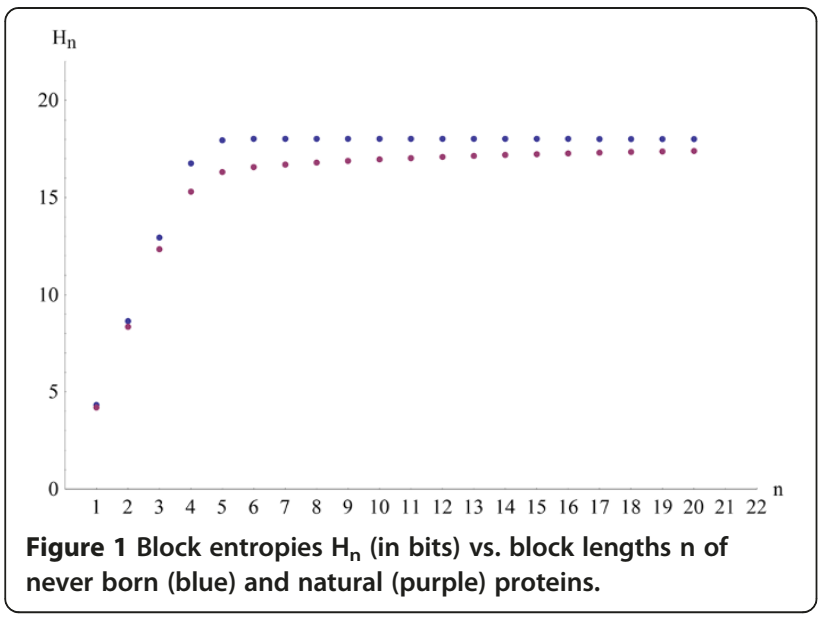

1996) and further intensively developed e.g. (Dewey 1996). In this short report we apply some of those ideas to Never Born proteins and we show that protein picking during evolution is closer to be a random/non-deterministic process. Our approach is strictly theoretical as (Strait \& Dewey 1996).

\section{Methods and results}

Natural proteins were randomly picked from UniProt database (The UniProt Consortium 2012). Never Born Proteins were generated with Random Blast tool (in both cases the number of sequences is 1250, in total around 400,000 amino acids, sequences lengths vary from 61 to around 1700 amino acids). Shannon entropies were calculated not only for every amino acid but also for blocks (block entropy (Papadimitriou et al. 2010)) of length from 2 to 20. Block entropy was calculated identically like Shannon entropy but probabilities refered to amino acid subsequences (blocks) of a specific length. Probabilities were normalized over occurrences in all sequences (the data and the scripts are available at http://www. cyfronet.pl/ myszonie/ent). The results are presented in Figure 1 and Table 1.

\section{Conclusions}

The plot (Figure 1) proves that the values of entropy for both groups of proteins are very close. It means that uncertainty or in other words a number of possible amino acid combinations, is almost the same. This indicates that natural protein sequences are random like Never Born Protein sequences. Moreover, relative entropy values show that encoding a natural protein sequence

Table 1 Relative entropies between never born and natural proteins

\begin{tabular}{cc}
\hline $\mathbf{D}_{\mathrm{KL}}\left(\mathbf{P}_{\mathrm{NBP}} \| \mathbf{Q}_{\mathrm{NP}}\right)$ & $\mathbf{D}_{\mathrm{KL}}\left(\mathbf{P}_{\mathrm{NP}} \| \mathbf{Q}_{\mathrm{NBP}}\right)$ \\
\hline 0.1554 & 0.1307 \\
\hline
\end{tabular}

using probability density of Never Born Proteins requires only a small excess of information (and vice versa). Summing up the inferences, protein selection during evolution is - in an information theory approach - closer to be a random process than deterministic one what is inline with (Jacob 1969; Jacob 2003; Weiss et al. 2000). There is still a doubt wheter that small difference does not play a key role.

\section{Competing interests}

The authors declare that they have no competing interests.

\section{Authors' contributions}

GS designed and carried out the research and drafted the manuscript. MJO supervised the research. Both authors read and approved the final manuscript.

\section{Acknowledgements}

The author would like to acknowledge Dr. Marcin Krol (Jagiellonian University, Poland) for the discussion and Prof. Fabio Polticelli (Roma Tre

University, Italy) for the Random Blast binaries.

Received: 20 December 2012 Accepted: 15 March 2013

Published: 30 April 2013

\section{References}

Chiarabelli C et al (2006) On the folding frequency in a totally random library of de novo proteins obtained by phage display. Chem Biodiver 3:840-859

De Lucrezia D et al (2012) Do natural proteins differ from random sequences polypeptides? Natural vs. random proteins classification using an evolutionary neural network. PLoS One 7:e36634

Dewey TG (1996) Algorithmic complexity of a protein. Phys Rev E 54:R39-R41

Evangelista $G$ et al (2007) Randomblast a tool to generate random never born protein sequences. Bio-Algorithms and Med-Systems 5:27-31

Jacob M (1969) On symmetry and function of biological systems. In: Engstrom A, Strondberg B (eds) Wiley Interscience, New York

Kullback BJ, Leibler RA (1951) On information and sufficiency. Ann Math Statist 22:79-86

Luisi PL (2003) Contingency and determinism. Phil Trans R Soc A 361:1141-1147

Munteanu CR et al (2008) Natural/random protein classification models based on star network topological indices. J Theor Biol 254:775-783

Papadimitriou C et al (2010) Entropy analysis of natural language written texts. Physica A 389:3260-3266

Shannon CE (1948) A mathematical theory of communication. Bell Labs Techn J 27:379-423

Strait BJ, Dewey TG (1996) The Shannon information entropy of protein sequences. Biophys J 71:148-155

The UniProt Consortium (2012) Reorganizing the protein space at the Universal Protein Resource (UniProt). Nucleic Acids Res 40:D71-D75

Weiss $O$ et al (2000) Information content of protein sequences. J Math Biol 206:379-386

doi:10.1186/2193-1801-2-200

Cite this article as: Szoniec and Ogorzalek: Entropy of never born

protein sequences. SpringerPlus 2013 2:200 